\title{
Response to neoadjuvant therapy with cisplatin in BRCA1-positive breast cancer patients
}

\author{
T. Byrski $\cdot$ T. Huzarski $\cdot$ R. Dent $\cdot$ J. Gronwald $\cdot$ \\ D. Zuziak · C. Cybulski · J. Kladny $\cdot$ B. Gorski $\cdot$ \\ J. Lubinski $\cdot$ S. A. Narod
}

Received: 20 June 2008/Accepted: 20 June 2008/Published online: 23 July 2008

(C) Springer Science+Business Media, LLC. 2008

\begin{abstract}
Background Ten patients with breast cancer and a breast cancer susceptibility gene 1 (BRCA1) mutation, who presented with stages I to III breast cancer between December 2006 and 2007, were treated with four cycles of neoadjuvant cisplatin, followed by mastectomy and conventional chemotherapy. Methods The excised breast tissue and lymph nodes were examined for the presence of residual disease. Results Pathologic complete response was observed in nine patients (90\%). Conclusions Platinum-based chemotherapy appears to be effective in a high proportion of patients with BRCA1-associated breast cancers. Clinical trials are now warranted to determine the optimum treatment for this subgroup of breast cancer patients.
\end{abstract}

\section{Keywords Cisplatin - BRCA1}

Abbreviations
$\begin{array}{ll}\text { BRCA1 } & \text { Breast cancer susceptibility gene 1 } \\ \text { DNA } & \text { Deoxyribonucleic acid } \\ \text { WHO } & \text { World Health Organization } \\ \text { BRCA2 } & \text { Breast cancer susceptibility gene } 2 \\ \text { PARP } & \text { Poly ADP-ribose polymerase }\end{array}$

T. Byrski · T. Huzarski · J. Gronwald · C. Cybulski · J. Kladny ·

B. Gorski · J. Lubinski

Pomeranian Medical University, Szczecin, Poland

R. Dent

Sunnybrook Odette Cancer Center, Toronto, ON, Canada

D. Zuziak

Regional Oncology Center, Bielsko-Biala, Poland

S. A. Narod $(\bowtie)$

Women's College Research Institute, 790 Bay Street, 7th Floor,

Toronto, ON, Canada M5G 1N8

e-mail: steven.narod@wchospital.ca

\section{Introduction}

Chemotherapy for breast cancer is often given prior to primary surgery (neoadjuvant chemotherapy) to decrease the size of the tumor in order that breast-conserving surgery can be performed, and to reduce the probability of lymph node involvement [1]. A further advantage of neoadjuvant therapy is that it helps to assess chemo-sensitivity to a particular agent; i.e. if the tumor does not diminish in size, then it is unlikely that the treatment will benefit the patient. In some cases, the breast cancer will regress completely, with no evidence of residual tumor, either in breast or the axilla (pathological complete response). In longitudinal studies, pathological complete response is predictive of prolonged disease-free survival and of overall survival [2].

The majority of breast cancer susceptibility gene 1 (BRCA1) mutation carriers diagnosed with breast cancer receive chemotherapy, because their tumors are typically high-grade [3], are estrogen-receptor negative [4] and exhibit rapid growth [5]. In two historical cohort studies, BRCA1-associated breast cancers appeared to be more sensitive to various forms of chemotherapy than nonhereditary tumors $[6,7]$. There is increasing evidence that deoxyribonucleic acid (DNA)-repair defects, which are characteristic of BRCA1-related cancers, confer sensitivity to particular systemic agents [8]. For example, in vitro studies of BRCA1-associated breast cancer cell lines show a high degree of sensitivity to agents that bring about interstrand cross-links, such as platinum-based drugs [9-12]. Shafee et al. studied mice which carried somatic mutations in BRCA1 and p53 in the breast tissue and who developed spontaneous breast cancers [13]. When treated with highdose cisplatin, $100 \%$ of 19 mice had a pathologic complete response. The same mice were refractory to doxorubicin. 
Platinum-based compounds have not been found to benefit the majority of breast cancer patients [14], but to date, no studies have investigated the role of platinum chemotherapy in the subgroup of BRCA1 mutation carriers. The objective of this study was to assess the frequency of complete pathologic response following neoadjuvant cis-platinum chemotherapy in women with breast cancer and a BRCA1 mutation.

\section{Materials and methods}

\section{Study design and entry criteria}

Patients were eligible for the study if they had a pathologically-proven diagnosis of invasive breast cancer by core biopsy or fine-needle aspiration biopsy and if they carried a pathogenic BRCA1 mutation. Eight patients were treated in Szczecin, Poland and two were treated in Bielsko-Biala. In these centers, genetic testing is offered to all women with incident breast cancer. BRCA1 testing was conducted for the three founder mutations seen in Poland, according to a previously-described protocol [15]. For the purpose of this study, genetic testing was expedited; in most cases the test result was available within five days of diagnosis. Written informed consent was obtained from each patient. The protocol was approved by the Research Ethics Review Board of the Pomeranian Medical Academy.

Patients with a previous diagnosis of breast cancer or other cancer were excluded (three patients). Patients who received prior chemotherapy were excluded (three patients). Patients with metastatic disease (stage IV) at presentation were excluded (one patient). One patient presented with primary breast cancer after a previous prophylactic bilateral mastectomy and was excluded.

No patient received any medication that affected renal function; none was pregnant or breastfeeding, and none had an active infection, symptomatic congestive heart failure, unstable angina pectoris, cardiac arrhythmia, or psychiatric illness. Patients were evaluated for renal function, liver function, and hematology prior to therapy. All were within normal limits.

Prior to platinum chemotherapy, the clinical and histologic features of the breast cancers were assessed. Tumor size was evaluated by clinical examination and by ultrasound, in some cases supplemented by mammogram. Histology was evaluated in nine cases by core biopsy and in one case by fine needle aspiration. For the nine cases that underwent core biopsy, grade, ER, PR, and ERBB2 status were evaluated by immuno-histochemistry. Immunohistochemical studies were not done for the patient who had a fine needle biopsy. Grade was evaluated in the core biopsy specimen using the Bloom-Richardson classification system.

\section{Treatment}

Cisplatin chemotherapy was administered at a dose of $75 \mathrm{mg} / \mathrm{m}^{2}$ every 3 weeks for four cycles. Each cycle was 21 days. Granulocyte colony-stimulating factor (G-CSF) was not given to any patient. Dexamethasone $(8 \mathrm{mg})$ was administered once daily for 3 days after chemotherapy. Ondansetron (Zophren ${ }^{\mathrm{TM}}$; GlaxoWellcome) was used for anti-nausea prophylaxis. Toxicity (nausea, vomiting, neutropenia) was assessed using the National Cancer Institute Common Toxicity Criteria after each cycle [16].

After cis-platinum chemotherapy, all patients were treated with mastectomy and axillary lymph node dissection. Surgery was followed by post-operative chemotherapy (four cycles of Adriamaycin and cyclophosphamide). Two patients received radiotherapy. No patient received Trastuzumab $\left(\right.$ Herceptin $^{\mathrm{TM}}$ ) or hormonal therapy.

\section{Response criteria}

Clinical response was considered complete if there was no evidence of the primary breast tumor according to the World Health Organization (WHO) criteria (total disappearance of the tumor in breast and lymph nodes) [17]. Clinical response was considered 'partial' if there was a reduction of $\geq 50 \%$ in the product of the two largest perpendicular diameters of the tumor. If the tumor area showed a reduction of $<50 \%$ or an increase of $>25 \%$ in the products of the largest diameter, then the patient was classified as a non-responder.

The primary endpoint of this study was pathologic complete response. Pathologic response was considered complete if there was no evidence of invasive breast tumor and the lymph nodes were negative. Pathology specimens were reviewed by two pathologists. If there was evidence of breast carcinoma in situ, but no evidence of invasive disease, this was still considered to be a pathologic complete response.

\section{Results}

\section{Patients}

From December 2006 to 2007, 10 patients were enrolled in the study. Patient characteristics are summarized in Table 1. All patients had a BRCA1 mutation; of these, eight $(80 \%)$ carried the common 5382insC founder mutation. The median age of diagnosis was 45 years (range 38-57 years). The median pre-treatment tumour size was 
Table 1 Baseline clinical characteristics of the study population $(N=10)$

\begin{tabular}{|c|c|c|}
\hline Characteristic & No. & $\%$ \\
\hline \multicolumn{3}{|l|}{ Age (years) } \\
\hline Mean & 46.7 & \\
\hline Range & $38-57$ & \\
\hline \multicolumn{3}{|l|}{ Type of BRCA1 mutation } \\
\hline 5382insC & 8 & 80 \\
\hline C61G & 2 & 20 \\
\hline \multicolumn{3}{|l|}{ Treating hospital } \\
\hline Szczecin & 8 & 80 \\
\hline Bielsko-Biala & 2 & 20 \\
\hline \multicolumn{3}{|l|}{ Clinical tumor stage } \\
\hline $\mathrm{T} 1(<2 \mathrm{~cm})$ & 5 & 50 \\
\hline $\mathrm{T} 2(\geq 2 \mathrm{~cm}$ to $\geq 5 \mathrm{~cm})$ & 4 & 40 \\
\hline $\mathrm{T} 3(>5 \mathrm{~cm})$ & 0 & 0 \\
\hline $\mathrm{T} 4$ & 1 & 10 \\
\hline \multicolumn{3}{|l|}{ Clinical nodal status } \\
\hline No & 7 & 70 \\
\hline N1 & 1 & 10 \\
\hline $\mathrm{N} 2$ & 2 & 20 \\
\hline N3 & 0 & 0 \\
\hline \multicolumn{3}{|l|}{ Bloom-Richardson grade } \\
\hline I & 0 & 0 \\
\hline II & 0 & 0 \\
\hline III & 9 & 90 \\
\hline Unknown & 1 & 10 \\
\hline \multicolumn{3}{|l|}{ Estrogen receptor levels } \\
\hline Positive & 0 & 0 \\
\hline Negative & 9 & 90 \\
\hline Missing & 1 & 10 \\
\hline \multicolumn{3}{|l|}{ Progesterone receptor levels } \\
\hline Positive & 0 & 0 \\
\hline Negative & 9 & 90 \\
\hline Missing & 1 & 10 \\
\hline \multicolumn{3}{|l|}{ HER2 status } \\
\hline 0 & 9 & 90 \\
\hline $1+$ & 0 & 0 \\
\hline $2+$ & 0 & 0 \\
\hline $3+$ & 0 & 0 \\
\hline Missing & 1 & 10 \\
\hline
\end{tabular}

$2.5 \mathrm{~cm}$ (range 1.0-6.5 cm). Clinically-positive lymph nodes were noted in three patients (30\%). Nine patients had immunohistochemical studies; all were negative for estrogen receptor, progesterone receptor and ERBB2 (i.e. were triple-negative).

Seven patients received the planned four cycles of cisplatin. Three patients stopped treatment after two cycles. One patient stopped due to side effects (nausea and vomiting) and two patients chose to stop treatment in order to
Table 2 Response to treatment

\begin{tabular}{lcr}
\hline Response & No. & $\%$ \\
\hline Clinical response & & \\
Complete response & 9 & 90 \\
Partial response & 1 & 10 \\
No change & 0 & 0 \\
Progressive disease & 0 & 0 \\
Pathologic response & & \\
Complete pathologic response & 9 & 90 \\
Partial reseponse & 1 & 10 \\
No response & 0 & 0 \\
Residual disease in breast & & 00 \\
None & 10 & 0 \\
$<1 \mathrm{~cm}$ & 0 & 0 \\
$1-3 \mathrm{~cm}$ & 0 & 0 \\
$4-9 \mathrm{~cm}$ & 0 & \\
$>9 \mathrm{~cm}$ & 0 & 00 \\
Number of lymph nodes positive & & 10 \\
0 & 9 & 0 \\
$1-3$ & 1 & \\
$4-9$ & 0 & 0 \\
$>9$ & & \\
\hline & &
\end{tabular}

expedite surgery. No patient experienced febrile neutropenia and no patient received G-CSF. No grades 3 or 4 anemia was observed.

After neoadjuvant cisplatin, no patient had clinical evidence of disease in the breast, but one patient had palpable lymph nodes (clinical response rate 90\%). The patient with a partial clinical response had received only two cycles of chemotherapy. All 10 patients then had a mastectomy and lymph node dissection. Nine patients achieved a complete pathological response (90\%) with no residual disease in the breast or the axilla (Table 2). The patient who did not achieve a clinical complete response (described above) had three (of 11) axillary nodes positive for tumour cells, but she had no residual disease in the breast. There was no evidence of ductal carcinoma in situ in any breast specimen.

\section{Discussion}

Our study indicates that a high proportion of women with BRCA1-associated breast cancers will respond to platinum-based chemotherapy. Four cycles of cis-platinum in the neoadjuvant setting resulted in a complete pathologic response in all seven patients who completed treatment. Only one patient did not achieve a pathologic complete response-she completed only two cycles of chemotherapy (of the three patients who received two cycles, two 
achieved pathologic complete response). Compliance was excellent and only one patient experienced significant toxicity, namely nausea and vomiting (a known side effect of cisplatin).

Despite the small sample size, this is the highest observed rate of pathological complete response for breast cancer for any treatment studied to date. Initial studies with neoadjuvant therapy with anthracycline and taxane combination chemotherapies achieved pathologic complete response (pCR) rates of $15-34 \%$ [18]. With the introduction of trastuzumab (Herceptin ${ }^{\mathrm{TM}}$ ) for HER2 positive patients, pCR rates have been observed as high as $68 \%$ in some studies [17].

Pathologic complete response is a good predictor of recurrence-free survival. Liedtke et al. [2] reviewed outcomes in 1,118 patients who received neoadjuvant chemotherapy (various agents) at MD Anderson Cancer Center from 1985 to 2004 . Overall, $15 \%$ of the patients experienced a pathologic complete response, including 57 of 255 patients (22\%) with triple-negative breast cancer. Of the 57 patients with triple negative breast cancer who experienced a pathologic complete response, the overall five-year survival rate was $94 \%$, and all deaths occurred in the 3 years following of treatment. The majority of BRCA1-associated breast cancers are triple-negative; in our study, all patients had triple-negative breast cancer (one was not tested). It is hoped that the survival experience of BRCA1-positive, triple-negative breast cancer patients with pathologic complete response to cisplatinum will be similar to that of the patients in the MD Anderson series. This will be the subject of future studies.

Platinum-based chemotherapy is the standard of care for patients with invasive ovarian cancer. Observational trials suggest that carriers of BRCA1 mutations with ovarian cancer may be more sensitive to the effect of platinumbased chemotherapy than non-carrier women [19]. Our study suggests that clinical studies of cisplatin might also be extended to BRCA1 carriers with other solid tumors, such as prostate and pancreatic cancer or to breast cancer susceptibility gene 2 (BRCA2) patients. There is also interest in evaluating the effect of platinum-based therapy in triple-negative breast cancer patients who do not harbor a BRCA1 mutation. It may be that the best use of platinumbased chemotherapy will be in combination with agents that potentiate the effects of DNA-damaging agents, such as the inhibition of enzymes involved in base-excision repair, e.g. Poly (ADP-ribose) polymerase (PARP) [20]. We believe that the homogeneity in response to cisplatin that we have observed is a reflection of the homogeneous etiology underlying the breast cancers. Our study confirms the potential benefit of tailoring therapy to specific subgroups of breast cancer patients.
Acknowledgements We would like to thank M. Siolek, M. Szwiec, D. Surdyka, O. Ashuryk, T. Debniak, R. Wisniowski and D. Sawka for helping to recruit patients to this study. We thank W. Domagala for the pathology review.

\section{References}

1. Gralow JR, Burstein HJ, Wood W et al (2008) Preoperative therapy in invasive breast cancer: pathologic assessment and systemic therapy issues in operable disease. J Clin Oncol 26:814819. doi:10.1200/JCO.2007.15.3510

2. Liedtke C, Mazouni C, Hess KR et al (2008) Response to neoadjuvant therapy and long-term survival in patients with triplenegative breast cancer. J Clin Oncol 10:1275-1281. doi:10.1200/ JCO.2007.14.4147

3. Lakhani SR, Jacquemier J, Sloane JP et al (1998) Multifactorial analysis of differences between sporadic breast cancers and cancers involving BRCA1 and BRCA2 mutations. J Natl Cancer Inst 90:1138-1145. doi:10.1093/jnci/90.15.1138

4. Foulkes WD, Metcalfe K, Sun P et al (2004) Estrogen receptor status in BRCA1- and BRCA2-related breast cancer: the influence of age, grade, and histological type. Clin Cancer Res 10:2029-2034. doi:10.1158/1078-0432.CCR-03-1061

5. Tilanus-Linthorst MM, Obdeijn IM, Hop WC et al (2007) BRCA1 mutation and young age predict fast breast cancer growth in the Dutch, United Kingdom, and Canadian magnetic resonance imaging screening trials. Clin Cancer Res 13:7357-7362. doi: 10.1158/1078-0432.CCR-07-0689

6. Robson ME, Chappuis PO, Satagopan J et al (2004) A combined analysis of outcome following breast cancer: differences in survival based on BRCA1/BRCA2 mutation status and administration of adjuvant treatment. Breast Cancer Res 6:R8-R17. doi: $10.1186 /$ bcr658

7. Rennert G, Bisland-Naggan S, Barnett-Griness O et al (2007) Clinical outcomes of breast cancer in carriers of BRCA1 and BRCA2 mutations. N Engl J Med 357:115-123. doi:10.1056/ NEJMoa070608

8. Kennedy RD, Quinn JE, Mullan PB, Johnston PG, Harkin DP (2004) The role of BRCA1 in the cellular response to chemotherapy. J Natl Cancer Inst 96:1659-1668

9. Quinn JE, Kennedy RD, Mullan PB et al (2003) BRCA1 functions as a differential modulator of chemotherapy-induced apoptosis. Cancer Res 6319:6221-6228

10. Bhattacharyya A, Ear US, Koller BH, Weichselbaum RR, Bishop DK (2000) The breast cancer susceptibility gene BRCA1 is required for subnuclear assembly of Rad51 and survival following treatment with the DNA cross-linking agent cisplatin. J Biol Chem 275:23899-23903. doi:10.1074/jbc.C000276200

11. Chabalier C, Lamare C, Racca C, Privat M, Valette A, Larminat F (2003) BRCA1 down-regulation leads to premature inactivation of spindle checkpoint and confers paclitaxel resistance. Cell Cycle 5:1001-1007

12. Zhou C, Smith JL, Liu J (2003) Role of BRCA1 in cellular resistance to paclitaxel and ionizing radiation in an ovarian cancer cell line carrying a defective BRCA1. Oncogene 22:23962404. doi:10.1038/sj.onc. 1206319

13. Shafee N, Smith CR, Wei S et al (2008) Cancer stem cells contribute to cisplatin resistance in BRCA1-p53-mediated mouse tumours. Cancer Res 68:3243-3250. doi:10.1158/0008-5472. CAN-07-5480

14. Decatris MP, Sundar S, O’Byrne KJ (2004) Platinum-based chemotherapy in metastatic breast cancer: current status. Cancer Treat Rev 4:53-81. doi:10.1016/S0305-7372(03)00139-7 
15. Górski B, Jakubowska A, Huzarski T et al (2004) A high proportion of founder BRCA1 mutations in Polish breast cancer families. Int J Cancer 110:683-686. doi:10.1002/ijc.20162

16. NCI National Cancer Institute (2003) Common terminology criteria for adverse events (CTCAE), version 3, June 10, 2004 update. National Cancer Institute, National Institutes of Health, Bethesda, MD

17. Richards MA, Smith IE, Dixon JM (1994) ABC of breast diseases. Role Syst Treat Prim Operable Breast Cancer BMJ 309:1363-1366

18. Buzdar AU, Ibrahim NK, Francis D et al (2005) Significantly higher pathologic complete remission after neoadjuvant therapy with trastuzumab, paclitaxel, and epirubicin chemotherapy. Results of a randomized trial in human epidermal growth factor receptor 2-positive operable breast cancer. J Clin Oncol 23:36763685. doi:10.1200/JCO.2005.07.032

19. Cass I, Baldwin RL, Varkey T, Moslehi R, Narod SA, Karlan BY (2003) Improved survival in women with BRCA-associated ovarian carcinoma. Cancer 97:2187-2195. doi:10.1002/cncr. 11310

20. Turner N, Tutt A, Ashworth A (2005) Targeting the DNA repair defect of BRCA tumours. Curr Opin Pharmacol 5:388-393. doi: 10.1016/j.coph.2005.03.006 